\title{
Real-time accurate rendering of color and texture of car coatings
}

\author{
Eric Kirchner (1), Ivo van der Lans (1), Pim Koeckhoven (1), Khalil Huraibat (2), Francisco M. Martínez-Verdú (2), Esther Perales (2), \\ Alejandro Ferrero (3), Joaquin Campos (3). \\ (1) AkzoNobel Technology Group Color; Sassenheim; the Netherlands; \\ (2) Department of Optics, University of Alicante, Alicante, Spain; \\ (3) Instituto de Óptica, Consejo de Investigaciones Cientificas (CSIC); Madrid, Spain.
}

\begin{abstract}
The digital representation of three dimensional objects with different materials has become common not only in the games and movie industry, but also in designer software, e-commerce and other applications. Although the rendered images often seem to be realistic, a closer look reveals that their color accuracy is often insufficient for critical applications. Storage of the angledependent color properties of metallic coatings and other gonioapparent materials demands large amounts of data. Apart from that, also rendering sparkle, gloss and other visual texture phenomena is still a subject of active research. Current approaches are computationally very demanding, and require manual ad-hoc setting of many model parameters.
\end{abstract}

In this paper, we describe a new approach to solve these problems. We combine a multi-spectral physics-based approach to make BRDF representation more efficient. We also account for the common loss in color accuracy due to the varying technical specifications of displays, and we correct for the influence from ambient lighting. The rendering framework presented here is shown to be capable of rendering sparkle and gloss as well, based on objective measurement of these properties. This takes out the subjective phase of manual fine-tuning of model parameters that is characteristic for many current rendering approaches.

A feasibility test with the new spectral rendering pipeline shows that is indeed able to produce realistic rendering of color, sparkle, gloss and other texture aspects. The computation time is small enough to make the rendering real-time on an iPad 2017, i.e. with low memory footprint and without high demands on graphic card or data storage.

\section{Introduction}

Computer Aided Design (CAD) has become a common tool in many industries, varying from automotive and architecture to furniture and interior design. It allows designers, manufacturers and customers to digitally visualize how future products would look based on choices for object shape and materials. By reducing or eliminating the need of physical mockup models, shorter development time and lower costs are obtained. It is therefore expected that the popularity of Computer Aided Design will further grow in the coming years. Also in many other industries where color plays an important role, the digitization of the color process will increase the demand for accurate rendering of materials [1][2][3].

Many different software packages and rendering engines are commercially available, producing rendered images that are often impressive, and well serve their users. For general applications well known examples are Maya and Keyshot. More specialized software are for example VRED (Autodesk), Spheron and Opticore, which are examples of rendering software mostly dedicated to the automotive market. For architects and designers, Revit is often used, in combination with rendering engines such as Mentalray (Nvidia), iRay, V-Ray, ART and A360. For complex materials, the input data for this rendering software could come from highend instruments such as the recent TAC7 (X-Rite) [4].
Apart from these commercial software packages, also academic software is available. Examples are from Integra [5], and algorithms from the University of Bonn [6][7].

While the images created by the available rendering software often suggest (and sometimes even claim) photorealistic quality, a visual comparison of these images with the physical objects they represent will show that they are often not realistic in terms of color accuracy [8]. For critical applications such as automotive design, the color accuracy of these images is not good enough [9]. Sparkle and other texture effects have proven to be "particularly difficult" to render [6], and therefore they are either absent or crudely represented in both commercial $\mathrm{CAD}$ and academic rendering software [7][8][10], even in reference textbooks [11][12][13]. Academic research has led to approaches for the rendering of metallic sparkle that achieve high-quality results [14][15], but these are computationally demanding, not applicable for real-time applications, and requiring manual setting of numerous parameters [6]. Materials with different gloss levels can be visualized in current rendering engines, using a number of standard models [11][12][13]. However, current rendering software is not able to utilize accurate measurement data on gloss of materials for accurately rendering this aspect of material appearance.

Another disadvantage from current $\mathrm{CAD}$ programs is that because of computational demands they require fast, expensive processors and/or graphical cards. This makes it impossible to produce live rendering on lighter laptops or tablet computers.

For typical applications such as the games and movie industries, these drawbacks from current rendering software are less important. Color accuracy with respect to physical objects is not an issue for both industries. A convincing representation of sparkle and other texture effects is also less important for the games and movie industry. Computational speed for rendering is no issue for the movie industry. For the games industry it seems to be acceptable that high end games with impressive graphics only run on high end PCs and game consoles.

However, for other industries there is a need for rendering techniques that overcome the problems just mentioned with current CAD solutions. Such techniques would be helpful for applications such as e-commerce and other industrial software. In this article, we introduce a new rendering pipeline that we developed to overcome the problems encountered with current CAD solutions. We will describe our research and results on developing truly realistic rendering methods that do provide high color accuracy, perceptually accurate representation of gloss, sparkle and other texture effects, and fast computation times even on typical tablet computers.

Here, we introduce a new approach to 3D rendering of complex materials such as gonio-apparent coatings, gloss, sparkle and other textured materials. Our method is unique in combining a number of advantages over existing rendering techniques, by (1) optimizing color accuracy by accounting for technical specifications of display and ambient light conditions, (2) further optimizing color accuracy by a fully spectral rendering pipeline, (3) also further optimizing color accuracy by applying a physically-based method for efficient BRDF interpolation of gonioapparent materials such as metallic coatings, (4) introducing accurate perception-based rendering of gloss, sparkle and other texture features, (5) being fast and computationally efficient enough to allow live rendering on tablet computers. 


\section{Framework}

The new approach was implemented by creating a dedicated framework for rendering. It is based on OpenGL 3.0 ES (Embedded Systems). This is an open source graphics library that provides the basic functionality of rendering.

As a hardware platform, we decided to not use high-end PC or graphic cards, to avoid developing solutions that only run well on high-end hardware. Therefore, we decided to develop the framework for an iPad (2017 edition) tablet computer. Since OpenGL is supported also on Android devices and Windows PCs, we expect no major problems for the portability of the framework to the other technology platforms.

\section{Spectral rendering pipeline}

Obviously, final images from rendering constitute of RGB images. In this respect, the new approach is not different from the technology used in commercial rendering software. However, in contrast to the methods used in that software, the new rendering pipeline uses a fully spectral approach. In our current implementation, up to the final stage when the resulting frame (image) is expressed in RGB, all calculations are conducted for different spectral bands. In this way, the rendering pipeline can be connected to spectral reflectance data from materials, which are measured with spectrophotometers. This data represents the full visible range of wavelengths, from 400 to $700 \mathrm{~nm}$, with a spectral resolution of $20 \mathrm{~nm}$, which indeed results in 16 different spectral bands. Spectral reflectance data are obviously provided as floating-point numbers.

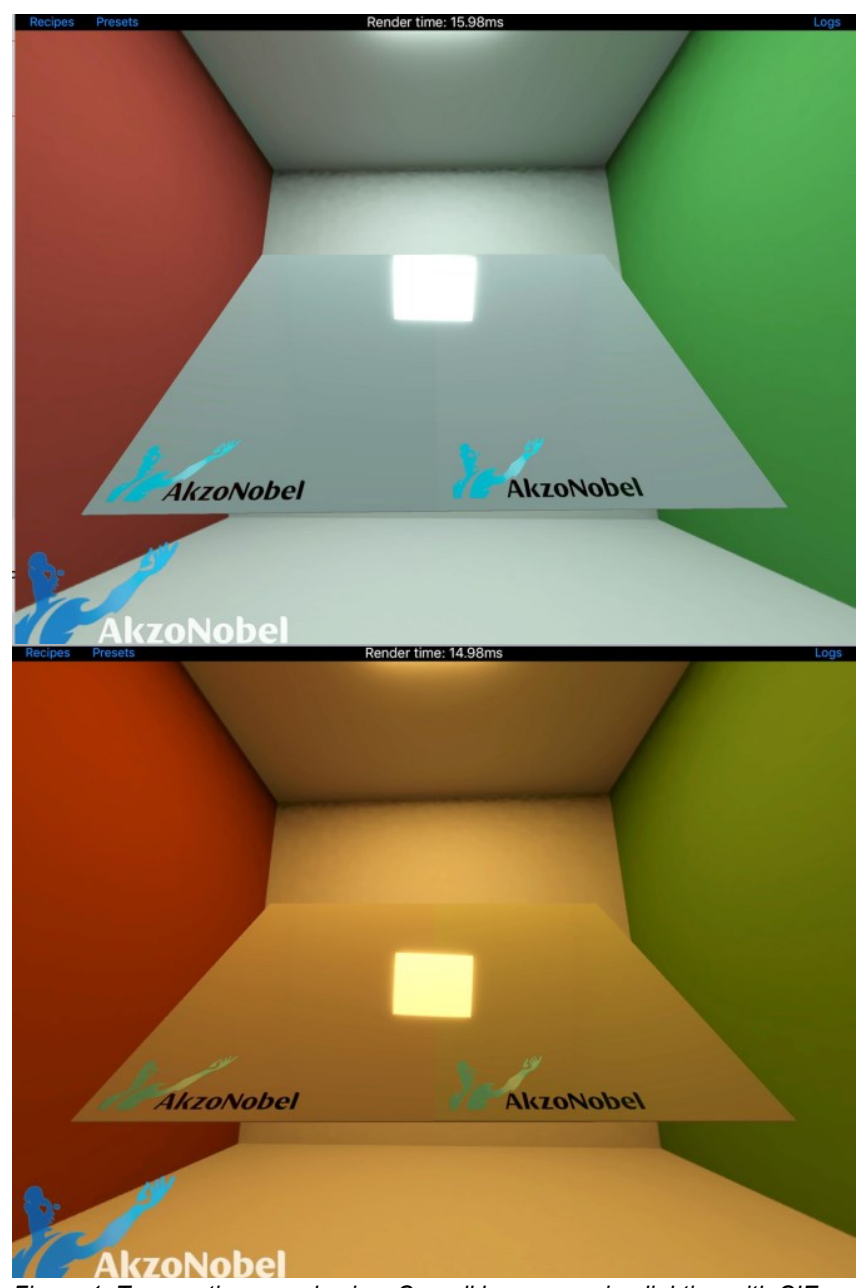

Figure 1. Two coating samples in a Cornell box, assuming lighting with CIE Standard Illuminants (a) D65 and (b) A as spectral power distributions. These two samples are metameric under D65, which is correctly reproduced because of the fully spectral rendering pipeline.
The result of this fully spectral rendering pipeline is that the color accuracy of the resulting frames (images) is improved with respect to images from common rendering software. The spectral rendering pipeline accounts for the spectral power distribution of light in the displayed environment. This is illustrated in Figure 1, where we show the rendering of two coating samples next to each other, as viewed in a simulated environment (Cornell box). The spectral reflectance data for the two coatings were measured with a spectrophotometer, and are used as input for the rendering pipeline. We took two coatings that are metameric with respect to each other: these two coatings show a good visual match under daylight (CIE Standard Illuminant D65), but under the so-called CIE Standard Illuminant A there is a visual mismatch. Figure 1a shows the result of rendering with D65, and there is a good visual color match between the two samples. In Figure 1b we have changed the spectrum of the light in the Cornell box to the A illuminant. Indeed, the rendered image of Figure $1 \mathrm{~b}$ clearly shows a color difference between the two samples.

Commercial rendering software would render this scene using three wavelength bands throughout the pipeline (R, G, B). As a consequence, the metameric effect shown in Figure 1 would not be visible in images created by commercial rendering software. Obviously, by manual adjustment of "the color" of the samples images like Figure $1 \mathrm{a}$ and $1 \mathrm{~b}$ could be created also with commercial rendering software, but this would be an ad-hoc solution. The new rendering pipeline is consistent in using spectrally correct calculations.

\section{Simulation of light booth}

For rendering an object in a lighting scene, we need to standardize this environment. The lighting environment for the rendering pipeline is specified in terms of the IES/EULUMDAT format. This enables the user to specify the luminous intensity distribution from lamps and reflective areas surrounding the object that needs to be rendered.

Apart from the well-known Cornell box as a simulated environment, we also developed a description of the light inside a light booth to test the new spectral rendering pipeline. For this, we selected the Byko-spectra effect light booth (BYK-Gardner). This light booth is particularly suitable for this investigation, since it was designed such as to optimize the visibility of sparkle and other texture effects in automotive coatings.

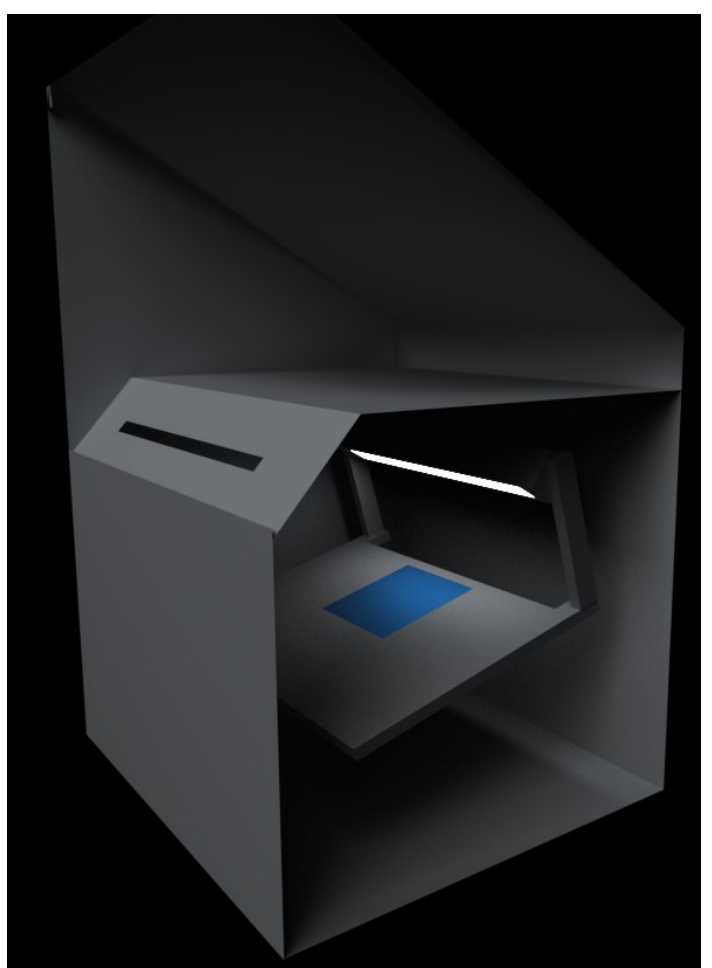

Figure 2. Rendering for an object inside a Byko-spectra effect light booth. 
Here, we used measurement data for the spectral power distribution and for the spatial distribution of luminous intensity inside the Byko-spectra effect light booth, as published before [16]. We used measurement data for luminance from 55 positions at the floor part of the light-booth, and measured all spatial dimensions and reflectance values for all materials inside the light booth. The light tubes inside the light booth were represented by $N$ spotlight sources (starting with $N=8$ and increasing until convergence is reached), each emitting light according to a Gaussian function in angle.

$$
\begin{aligned}
& E(x, y, \theta)=\sum_{i=1}^{N} \frac{I(\theta)}{d^{2}} \\
& I(\theta)=I_{0} \exp \left[-\theta^{2} / 2 \sigma^{2}\right]
\end{aligned}
$$

where $d$ is the distance between light spot and measurement point. The illuminance $I_{0}$ and Gaussian width $\sigma$ of these functions were optimized as best fit parameter values, thereby forming a luminance model of the light tubes inside the light booth. We used those parameter values to calculate numerical values to populate the IES/EULUMDAT file as input for describing the light environment. As a result, we are now able to render objects as they would appear inside a Byko-spectra effect light booth. Figure 2 shows an example of this rendering.

\section{Account for display specifications \& ambient lighting}

In common rendering software, the calculation of RGB images utilizes the device-independent sRGB method. This method does not take the technical specifications into account of the electronic display that is used to show the rendered images.

Electronic displays vary considerable in terms of the displayed colors, even when using the same RGB images as input. As an example, researchers report that the display of the iPad Air 2 tablet computer becomes "slightly too blue" when showing pure white [17][18]. When showing dark images, and also for bright ambient light conditions, the same display shows a blue cast, possibly due to the antireflective coating on the display [18]. By using the device-independent sRGB method, rendered images will show this blue cast.

Recently, we developed an alternative to the SRGB method, that does take into account the technical specifications of a display [19][20]. As an example, this method does take away the blue cast reported for the iPad Air2 tablet computer display. The new method is called the Mobile Display Characterization and Illumination Model (MDCIM). It not only accounts for variations between display characteristics, but it also corrects for the influence from the intensity (illuminance) of ambient light. As is well known, under highly intense ambient light, colors appear to be more saturated than under dim lighting conditions.

In the rendering pipeline reported in the current article, we implemented this new MDCIM model. It is applied in the final stage of calculating a frame (image). Because of this aspect, the rendered frames are more color accurate than those from previous rendering frameworks, as it corrects for specific display characteristics and local ambient lighting. We implemented dithering as a further means to achieve higher color accuracy.

The issues mentioned in this section are well known. In current rendering software, they may be avoided by using a color calibrated display and by assessing objects under standardized lighting conditions, such as a light booth with prescribed illuminance level of 1000 lux. By implementing the MDCIM model in the new rendering pipeline, we obtain a solution that can be applied more generally. For example, we apply it to an iPad tablet computer, for which color calibration is hard to accomplish.

\section{Physics-based BRDF Interpolation}

The color of effect coatings and other gonio-apparent materials strongly varies with angles of viewing and incidence. It is important to take this into account in order to obtain accurate rendering of objects covered by these materials. First steps towards this goal have been published by other researchers. For example, Shimizu and Meyer reported on developing computer aided color design software that renders metallic coatings $[21][22][10]$. In that approach, the dependence of lightness on viewing and incidence angle was modeled by a polynomial function.

In the new rendering framework, we use a more refined approach by representing the full Bidirectional Reflectance Distribution Function (BRDF). In other words, instead of only considering lightness we represent the dependence of all reflectance values on viewing and incidence directions. We use the following two different ways of representing the BRDF in the spectral rendering pipeline.

Firstly, we use a mathematical model that results from a Principal Component Analysis of the BRDF, by separating geometric variables (angles of viewing and incidence) from the spectral variable (wavelength) [23]. The resulting mathematical expression has been shown to accurately represent the full BRDF of an interference pigment, which makes it very likely that it will also be useful for metallic coatings. We note that there also other mathematical models have been proposed in the past for representing BRDF functions [11][12][13], which can be implemented in the new rendering pipeline.

Secondly, we have developed new concepts for describing the angular variation of the reflectance of gonio-apparent materials. Instead of using the common angles for viewing and incidence, we have presented a physical analysis that indicates that flake-based parameters are to be preferred [24]. The reflectance properties of an effect coating are determined primarily by the effect flakes inside the coating. This makes it preferable to use parameters with respect to the local normal vectors to these flakes. It has already been shown that these flake-based parameters are much more effective for representing BRDF functions than the common viewing and illumination angles [25][26][27][28][29].

By using an accurate interpolation of the $\mathrm{BRDF}$, the new rendering pipeline is computationally much faster than when the full measured BRDF data need to be used, as is the case for some existing rendering approaches [30]. For example, the TAC7 instrument that was recently launched by X-Rite is capable of producing impressive rendering of e.g. the sparkle of metallic coatings, but it has the disadvantage of needing large data files (typically gigabytes per sample), making it impossible to handle thousands of samples on a common platform such as an iPad [4]. A disadvantage of the current interpolation could be a lower color accuracy. Therefore this aspect will need to be investigated in future work.

\section{Perception-based rendering of sparkle and gloss}

For computer rendering of materials with gloss levels varying from high gloss to matt, a range of reflectance models have been proposed over the years [11][12][13]. Current rendering software often utilizes one or more of these models, such as those from Phong [31], Cook and Torrance [32], Ashikhmin et al. [33]. The development of this type of models to render sparkle and/or gloss effects is still an active field of research $[14][15][34][35][36]$. These models are usually derived from assuming a material surface consisting of microfacets, and parametrizing the resulting contributions from diffuse and specular reflectance. This assumption may indeed be a good physical description for the physical cause of gloss effects. Therefore, also in the rendering pipeline introduced in this article we have the possibility of using one or more of these standard models.

However, there is another aspect of the rendering of gloss where the new rendering method will go beyond the capabilities of current rendering software. The user of current software chooses numerical values for a number of model parameters, which ultimately lead to a change in material (gloss) appearance. This choice of model parameter values is typically done in an ad-hoc way, by visually judging the gloss aspect of the rendered image. In some rendering software, the user selects the appropriate gloss level by pre-defined categories, such as high gloss, satin, matte, etc.

The new rendering framework is an improvement over crude subjective gloss characterization, by allowing the use of data from a gloss meter such 
as the Micro-Tri-gloss meter (BYK-Gardner, Geretsried, Germany). We will separately report on how we carried out a perception-based investigation to optimize the conversion from tri-gloss measurement data to gloss model parameters. As a consequence, the rendering engine produces fully objective gloss rendering (cf. Figure 3a).

We took a similar approach to rendering sparkle and other texture effects. In the past, we already reported on the development of different perceptionbased algorithms for rendering sparkle and graininess (diffuse coarseness) of car paints [37][38][39]. A similar algorithm was also implemented in the new rendering engine (Figure $3 b$ ). In the near future we will finetune the conversion from measured texture data to the model parameters for rendering sparkle and graininess. In this way, we will be able to utilize data on texture measured with commercial instruments, such as the BYK-Mac i (BYK-Gardner) and the recently introduced MA-T6 multi-angle spectrophotometer (X-Rite).

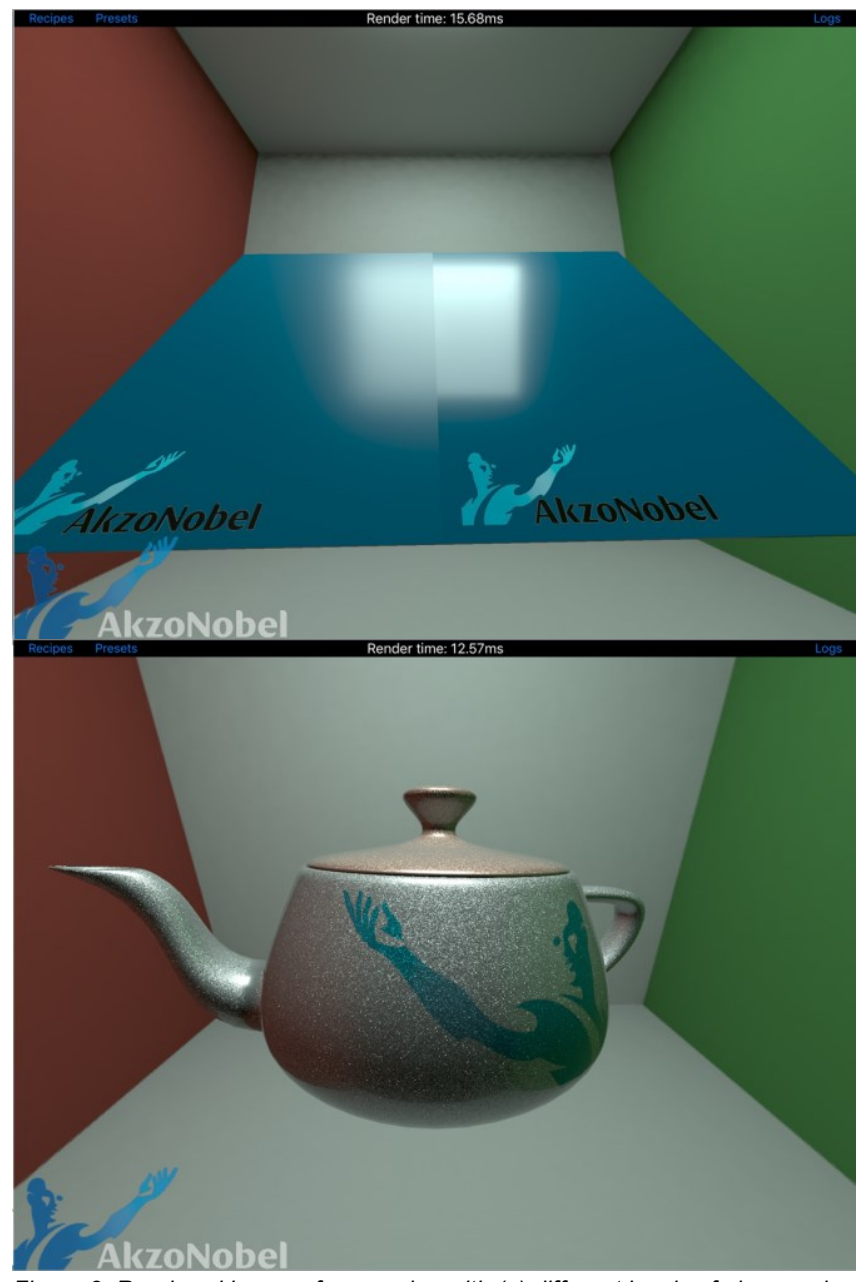

Figure 3. Rendered images for samples with (a) different levels of gloss and (b) sparkle

\section{Computationally fast, low demands on data}

The code of the spectral rendering pipeline was optimized to make it computationally fast. As a result, even on the graphical processor of a tablet computer like the iPad Air 2 or the iPad Air (2017 edition), and even for rendering models of complex objects such as cars that require hundreds of polygons, the rendering typically takes $30 \mathrm{~ms}$ per frame. This is more than sufficient for common industrial applications.

We note that most commercial rendering software requires relatively highend computing power, for example in the form of high-end graphical cards and a PC that goes beyond normal office requirements.

\section{Conclusions}

In this article, we present a new spectral pipeline for rendering gonioapparent materials such as effect coatings. We have described how we are able to achieve a number of advantages over current rendering software: (1) higher color accuracy by taking the technical characteristics of a display and the ambient light conditions into account, (2) by making the rendering pipeline fully spectral, (3) and by utilizing a recently developed physicsbased and efficient method for interpolating BRDF data of gonio-apparent materials such as metallic coatings, (4) developing perception-based rendering of gloss, sparkle, graininess and other aspects of texture, (5) allowing fast computation and live rendering even on tablet computers.

As described in this article, the new spectral rendering pipeline allows using measurement data on gloss and sparkle. Current rendering software typically requires the user to make subjective choices on rendering these phenomena. Many rendering software solutions do not even include rendering of metallic sparkle. In the few cases where sparkle rendering is possible, it requires handling very large data files [40][7] and/or high-end computation power and high-end displays [41][3].

\section{Acknowledgements}

This study was also supported by the Spanish Ministry of Economy and Competitiveness under grant no. DPI2011-30090-C02-02 and the European Union. Khalil Huraibat would also like to thank the Ministry of Economy and Competitiveness for his pre-doctoral fellowship grant (FPI BES- 2016077325). The authors are also grateful to EMRP for funding the project "Bidirectional Reflectance Definitions". The EMRP is jointly funded by the EMRP participating countries within EURAMET and the European Union.

\section{References}

[1] R. Martín, M. Weinmann, M.B. Hullin, "Digital transmission of subjective material appearance", Jour. WSCG 25, no. 2, pp. 123-132, 2017.

[2] Pantone Live, https://www.pantone.com/products/digitalapps/pantonelive-design.

[3] DMIX Cloud, https://www.deutschesmodeinstitut.de/kopie-von-diedmix-cloud.

[4] S. Mezbach, M. Weinmann, R. Klein, "High quality multi-spectral reflectance acquisition with X-Rite TAC7”, Proceedings of the Eurographics workshop on Material Appearance Modeling, 2017.

[5] http://www.integra.jp/en/products/lumicept, 2016.

[6] T. Golla and R. Klein, "An efficient statistical data representation for real-time rendering of metallic effect car paints", Proceedings of the International Conference on Virtual Reality and Augmented Reality EuroVR (Laval, France), pp. 51 - 68, 2017.

[7] M. Rump, G. Müller, R. Sarlette, D. Koch, R. Klein, "Photo-realistic Rendering of Metallic Car Paint from Image-Based Measurements", Eurographics/Computer Graphics Forum 27 (Crete, Greece), pp. 527536, 2008.

[8] J. Günther, T. Chen, M. Goesele, I. Wald and H.P. Seidel, "Efficient acquisition and realistic rendering of car paint" in G. Greiner, J. Hornegger, H. Niemann and M. Stamminger, eds., Proceedings of Vision, Modeling and Visualization (Erlangen, Germany), pp. 487494, 2005.

[9] J.A. Ferwerda, S.H. Westin, R.C. Smith and R. Pawlicki, "Effects of rendering on shape perception in automobile design". Proceedings ACM Symposium on applied perception in Graphics and Visualization (Los Angeles, US), pp. 107- 114, 2004.

[10] C. Shimizu, G.W. Meyer, "A computer aided color appearance design system for metallic car paint", J. Imaging Sci. and Technol. 59, 0303403-1-030403-10, 2015. 
[11] M. Pharr, W. Jakob and G. Humphreys, "Physically Based Rendering: From theory to Implementation", Morgan Kaufmann Publishers, third edition, 2016.

[12] M. Haindl and J. Filip, "Visual texture", Springer, 2013.

[13] J. Dorsey, H. Rushmeier ad F. Silion, "Digital Modeling of Material Appearance", Morgan Kaufmann Publishers, 2008.

[14] W. Jakob, M. Hasan, L.Q. Yan, J. Lawrence, R. Ramamoorthi, S. Marschner, "Discrete stochastic microfacet models", ACM Transactions on Graphics 33, 115, 2014.

[15] L.Q. Yan, M. Hasan, W. Jakob, J. Lawrence, S. Marschner, R. Ramamoorthi, "Rendering Glints on High-Resolution NormalMapped Specular Surfaces", ACM Transactions on Graphics 33, 116, 2014.

[16] F.M. Martínez-Verdú, E. Perales, V. Viqueira, E. Chorro, F.J. Burgos, J. Pujol, "Comparison of colorimetric features of some current lighting booths for obtaining a right visual and instrumental correlation for gonio-apparent coatings and plastics", Proceedings of the CIE Conference Lighting Quality and Energy Efficiency (Hangzhou, China), pp. 692 - 705, 2012.

[17] D. Schmidt, Apple iPad Air 2 (A1567/128 GB/LTE) Tablet Review, http://www.notebookcheck.net/Apple-iPad-Air-2-A1567-128-GBLTETablet-Review.129396.0.html.

[18] R. M. Soneira, "iPad 2014 display technology shoot-out," http://www.displaymate.com/iPad6_ShootOut.htm, 2014.

[19] E. Kirchner, I. van der Lans, F. Martínez-Verdú, E. Perales, "Improving color reproduction accuracy of a mobile liquid crystal display". J. Optic. Soc. Am. A 34, pp. 101-110, 2017.

[20] E. Kirchner, I. van der Lans, E. Perales, F. Martínez-Verdú. "Improving color reproduction accuracy of an OLED-based mobile display". Color Res. Appl. pp. 34-46, 2018.

[21] C. Shimizu, G.W. Meyer, J.P. Wingard, "Interactive Goniochromatic Color Design", in Proceedings of IS\&T/SID's Eleventh Color Imaging Conference (Springfield, VA) pp. 16-22, 2003.

[22] C. Shimizu, G.W. Meyer, "Color styling tools", Proceedings of the IS\&T/SID Eighteenth Color Imaging Conference (Springfield, VA), pp. 272279,2010 .

[23] A. Ferrero, A. Rabal, J. Campos, A. Pons, M. Luisa Hernanz, "Variables separation of the spectral BRDF for better understanding color variation in special effect pigment coatings", J. Optic. Soc. Am. A 29, pp. 842-847, 2012.

[24] E.J.J. Kirchner and W.R. Cramer, "Making sense of measurement geometries for multi-angle spectrophotometers", Color Res. and Appl. 37, pp. 186-198, 2012.

[25] E. Kirchner, I. van der Lans, A. Ferrero, J. Campos, F.M. MartínezVerdú, E. Perales, "Fast And Accurate 3D Rendering Of Automotive Coatings", Proceedings of the Twenty-third Color and Imaging Conference (Darmstadt, Germany), pp.154-160, 2015.

[26] A. Ferrero, E. Perales, A.M. Rabal, J. Campos, F.M. MartínezVerdú, E. Chorro and A. Pons, "Color representation and interpretation of special effect coatings", J. Optic. Soc. Am. A 31, pp. 436 - 447, 2014.

[27] A. Ferrero, B. Bernad, J. Campos, E. Perales, J.L. Velázquez, F.M. Martínez-Verdú, "Color characterization of coatings with diffraction pigments", J. Optic. Soc. Am. A 33, pp. 1978 - 1988, 2016.
[28] N. Rogelj, M.K. Gunde, "Goniospectrometric space curve for coatings with special effect pigments", Appl. Opt. 55, pp. 122-132, 2018.

[29] C. Strothkämper, K.O. Hauer, A. Höpe, "How to efficiently characterize special effect coatings", J. Optic. Soc. Am. A 33, pp. 18, 2018.

[30] C.M. Seubert, M.E. Nichols, C. Kappauf, K. Ellwood, M. Shtein, M.D. Thouless, "A hybrid ray-wave optics model to study the scattering behavior of silver metallic paint systems", J. Coat. Technol. Res. 15, pp. 471-480, 2018.

[31] B.T. Phong, "Illumination for computer generated pictures", Communications of the ACM 18, pp. 311-317, 1975.

[32] R.L. Cook and K.E. Torrance, "A reflectance model for computer graphics", ACM Transactions on Graphics 1, pp. 7-24, 1982.

[33] M. Ashikhmin, S. Premoze, P.S. Shirley, "A microfacet-based BRDF generator", in Proceedings of ACM SIGGRAPH Computer Graphics Proceedings, Annual Conference Series, pp. 65-74, 2000.

[34] S. Ergun, S. Önel, A. Ozturk, "A General Micro-flake Model for Predicting the Appearance of Car Paint", Proceedings of the Eurographics Symposium on Rendering - Experimental Ideas \& Implementations (Dublin, Ireland), E. Eisemann and E. Fiume (Editors) pp. 65-71, 2016.

[35] P. Lecocq, A. Dufay, G. Sourimant, J.E. Marvie, "Analytical approximations for real-time area light shading", IEEE Transactions on Visualization and Computer Graphics 23, pp. 1-14, 2017.

[36] B. Walter, S. Marschner, H. Li and K. Torrance, "Microfacet Surfaces for Refraction through Rough Surfaces" in Proceedings of the Eurographics Symposium on Rendering (Grenoble, France), pp. 195206, 2007.

[37] I.B.N. Van der Lans, E. Kirchner, A. Half, "Accurate appearancebased visualization of car paints", Proceedings of the Sixth European Conference on Colour in Graphics, Imaging and Vision (Amsterdam, the Netherlands) pp. 17-23, 2012.

[38] A. Ferrero, J. Campos, A.M. Rabal and A. Pons, "A single analytical model for sparkle and graininess patterns in texture of effect coatings", Opt. Express 21, 26812, 2013.

[39] A. Ferrero, " Definition of a measurement scale of graininess from reflectance and psychophysical measurements", Optics Express (accepted).

[40] J. Gu, C.I. Tu, R. Ramamoorthi, P. Belhumeur, W. Matusik, S. Nayar, "Time-varying surface appearance: acquisition, modeling and rendering”, Proceedings of the SIGGRAPH'06, (Boston, Mass.) pp. 762-771, 2006

[41] M.S. Ellens, F. Lamy, "From color to appearance in the real world", in Proceedings of SPIE Vol. 8291, Human Vision and Electronic Imaging XVII; (Burlingame, California) 82910B, 2012.

\section{Author Biography}

Eric Kirchner received his BS in physics from the University of Utrecht (1989) and his PhD in theoretical chemistry from the Free University in Amsterdam, the Netherlands (1993). Since 2000 he has worked in the Color Technology group at AkzoNobel in Sassenheim, the Netherlands. His work has focused on the accurate displaying, measurement and paint formulation of color, patterns and texture of paint materials. 


\section{JOIN US AT THE NEXT EI!}

IS\&T International Symposium on

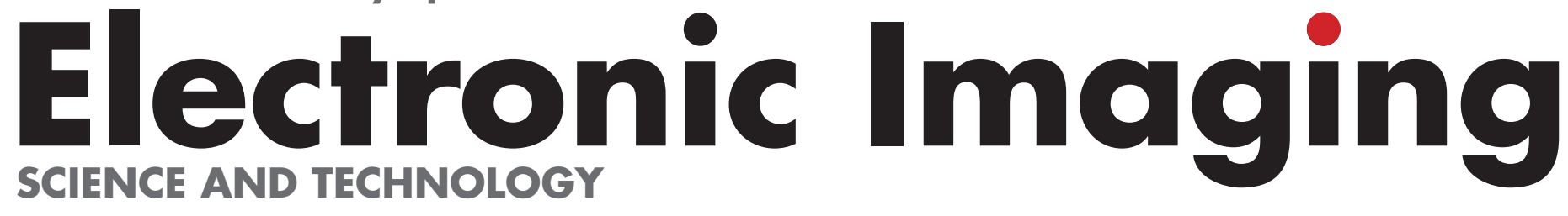

Imaging across applications ... Where industry and academia meet!
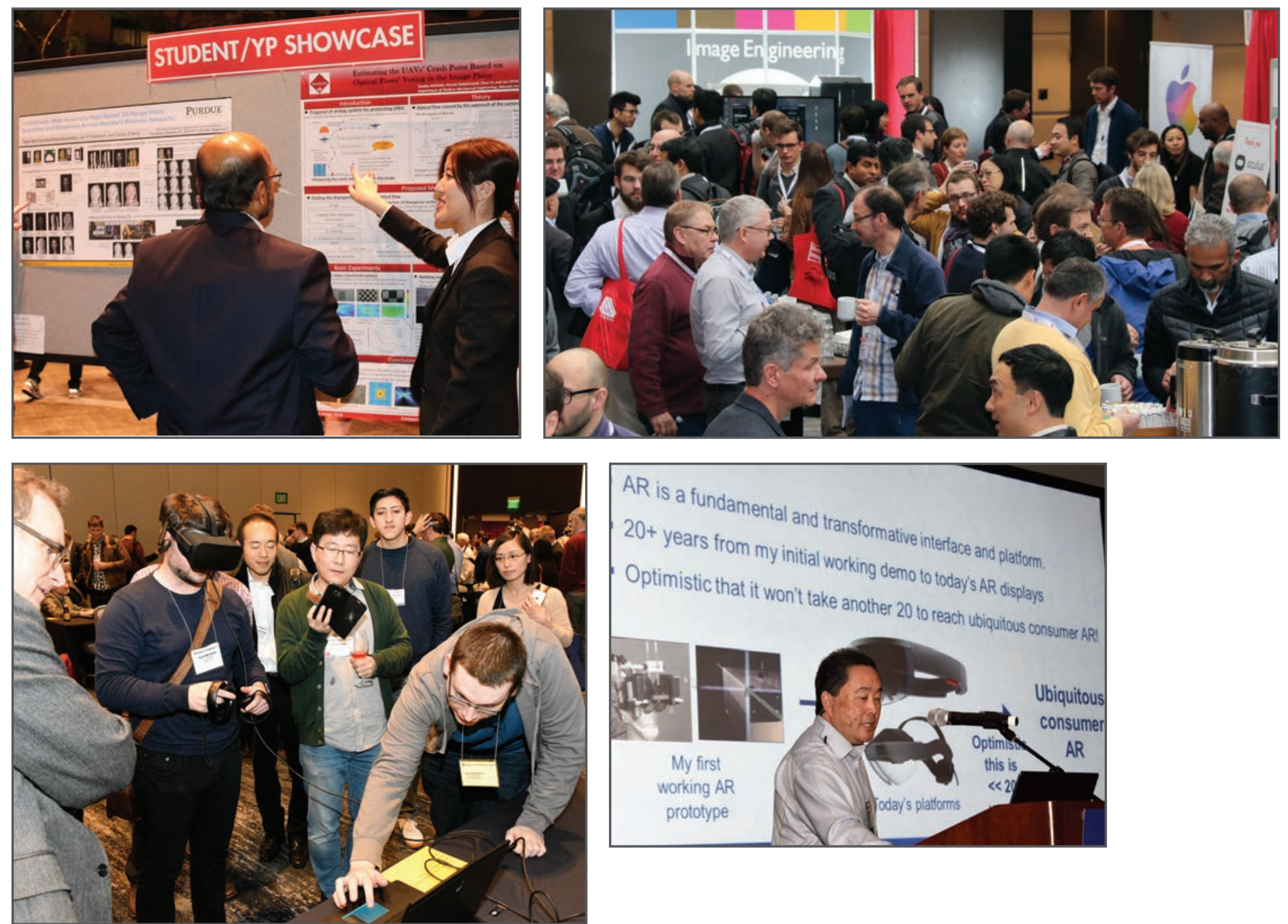

- SHORT COURSES • EXHIBITS • DEMONSTRATION SESSION • PLENARY TALKS •

- INTERACTIVE PAPER SESSION • SPECIAL EVENTS • TECHNICAL SESSIONS •

www.electronicimaging.org

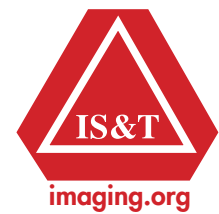

